\title{
Un anthropologue dans la cité
}

Hommage à Mathieu Hilgers

Pierre-Joseph Laurent et Jacinthe Mazzocchetti

\section{OpenEdition \\ Journals}

Édition électronique

URL : http://journals.openedition.org/anthropodev/360

DOI : 10.4000/anthropodev.360

ISSN : 2553-1719

Éditeur

APAD - Association pour l'anthropologie du changement social et du développement

Édition imprimée

Date de publication : 1 septembre 2015

Pagination : 15-23

ISBN : 979-10-93476-02-5

ISSN : 2276-2019

\section{Référence électronique}

Pierre-Joseph Laurent et Jacinthe Mazzocchetti, « Un anthropologue dans la cité », Anthropologie \& développement [En ligne], 42-43 | 2015, mis en ligne le 01 décembre 2016, consulté le 15 septembre 2020. URL : http://journals.openedition.org/anthropodev/360

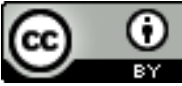

La revue Anthropologie \& développement est mise à disposition selon les termes de la Licence Creative Commons Attribution 4.0 International. 


\title{
Un anthropologue dans la cité. Hommage à Mathieu Hilgers
}

\author{
Pierre-Joseph Laurent et Jacinthe Mazzocchetti
}

\begin{abstract}
Cet hommage à Mathieu Hilgers retrace le parcours et les travaux d'un anthropologue qui a marqué sa discipline. L'anthropologie politique lui doit des débats importants et novateurs autour des notions de semi-autoritarisme, de collectif d'appartenance et d'autochtonie, des notions qui résonnent aujourd'hui avec force dans l'analyse de l'actualité africaine. Dans le champ de l'anthropologie de la ville, il s'illustre à travers sa réflexion sur les conditions méthodologiques d'une ethnologie des villes moyennes. Son apport à la connaissance du Burkina Faso, aux études africaines et à notre discipline est et restera significatif.

This tribute to Mathieu Hilgers retraces the career and work of an anthropologist who made his mark on the discipline. Political anthropology is indebted to him for important and pioneering debates on the notions of semi-authoritarianism, collectivity of belonging and autochthony, notions that resonate strongly today in the analysis of current events in Africa. In the field of urban anthropology, he distinguished himself through his reflections on the methodological prerequisites for an anthropology of midsized towns. His contribution to the knowledge of Burkina Faso, to African Studies and to our discipline, is significant and will also remain so.
\end{abstract}

Mathieu Hilgers nous a quitté le 28 février 2015, une perte incommensurable pour ses proches, nous pensons ici tout particulièrement à sa compagne Géraldine André, qui fut elle aussi l'une de nos collègues; mais également, comme l'énonçaient les très nombreux messages reçus lors de l'annonce de sa disparition brutale, une perte incommensurable pour la pensée sur et avec l’Afrique.

Licencié en philosophie et en sociologie, docteur en anthropologie, professeur d'anthropologie à l'université libre de Bruxelles (depuis 2010), Mathieu Hilgers fut membre du réseau APAD. Tout d'abord en tant que 
doctorant et, à partir de 2007, en tant que membre actif du Bureau, suite au colloque de l'APAD organisé conjointement par l'université catholique de Louvain (où il fit ses études) et le musée de Tervuren. II était sur la scène internationale connu et reconnu depuis quelques années déjà pour les débats scientifiques qu'il a initiés ainsi que pour la très haute qualité de ses publications; parmi lesquelles, de nombreuses contributions relatives au travail de Pierre Bourdieu (Hilgers et Mangez, 2014 ; Hilgers, 2007, 2009a et 2013a ; etc.). Remarquable également le cycle d'articles écrits sur la question du néolibéralisme, proposant à la fois une synthèse des réflexions en cours en anthropologie et un débat empiriquement ancré à partir de l'Afrique de l'Ouest, en dialogue notamment avec Loïc Wacquant (Hilgers, 2010, 2012 et 2013b). Parmi les articles publiés par Mathieu, à noter également le débat engagé avec Peter Geschiere autour de la question de l'autochtonie (Hilgers, 2011a, 2011b et 2011c) ; discussion reposant une fois encore sur une connaissance fine de la littérature afférente, ainsi que sur ses propres enquêtes de terrain en Afrique de l'Ouest, principalement au Burkina Faso et, ces dernières années, au Ghana. Ayant surtout eu l'occasion de collaborer avec Mathieu durant les années de sa thèse de doctorat et de post-doctorat, nous avons choisi de mettre ici à I'honneur ses travaux en africanistique, et plus précisément d'anthropologie politique, d'une part, et d'anthropologie de la ville, d'autre part.

Lorsque Mathieu Hilgers et Jacinthe Mazzocchetti publièrent en 2010 Révoltes et oppositions dans un régime semi-autoritaire, le cas du Burkina Faso, ouvrage issu d'un colloque organisé sous leur direction scientifique à l'université catholique de Louvain (en 2006), ils ignoraient tout de son destin. Diffusé au Burkina Faso, photocopié, lu, discuté, commenté dans les médias, l'ouvrage connaîtra un franc succès au pays des " Hommes intègres " : il entrait en résonance avec les aspirations d'une partie de la population. Avec d'autres facteurs certes, ce travail scientifique de premier plan contribua à clarifier le fonctionnement d'un régime "semiautoritaire ", avec son apparente liberté et le développement des espaces publics, couplés à la stabilité et à la longévité du régime. Bien avant la médiatisation progressive des crises de 2008, 2011 et 2014, le pari de l'ouvrage reposait sur la volonté de décrire la complexité du jeu politique burkinabè. Si comme l'énonçait Mathieu, en référence à Bourdieu, le sociologue et l'anthropologue arrivent généralement "quand la fête est 
finie, le rideau tombé, les lumières médiatiques éteintes ", si la temporalité entre les résultats de la recherche et l'état du monde implique toujours un décalage, rigoureux dans les analyses proposées et prospectif dans les perspectives esquissées, l'ouvrage annonçait la révolte populaire d'octobre 2014 qui mettra fin au régime de Blaise Compaoré et ouvrira une période de transition. Révolte populaire qui, pour les auteurs, n'avait cependant rien d'improvisé tant la soif de changement, à la fois étouffée et avivée par le contexte de semi-autoritarisme, la montée des tensions et des protestations, mais aussi l'apparition de nouveaux groupes dans la société civile, tel le Balai citoyen, étaient visibles ces dernières années. Plus encore, désormais, la révolution burkinabè agit comme une épée de Damoclès sur les dirigeants du continent africain tentés par une révision constitutionnelle pour se maintenir au pouvoir et briguer un " troisième mandat ", pressés par un entourage avide et des intérêts nationaux et internationaux. En Afrique noire, là où la population élève la voix pour protester, le Burkina Faso est cité en exemple, en RDC, au Congo-Brazzaville, au Cameroun, au Burundi, au Togo... Alors que de nombreuses élections sont prévues sur le continent dans les prochains mois, un vent de révolte souffle : I'information circule mieux et une jeunesse à l'écoute entend revendiquer un autre futur.

Pour les auteurs de l'ouvrage, dans la suite des travaux de Marina Ottaway (2003), les régimes " semi-autoritaires " se caractérisent par une tension : pour exister, ils doivent nourrir et rendre institutionnellement possibles les espérances politiques qu'ils cherchent à neutraliser. Cependant, malgré le contrôle qu'exerce le pouvoir dans de tels régimes, les changements institutionnels transforment les mentalités, façonnent une meilleure connaissance des mécanismes et des rouages politiques et, dans certains cas, de plus grandes velléités d'opposition et, donc, un potentiel subversif qui peut avoir des effets sur l'espace politique (voir également André et Hilgers, 2009). Ainsi, les études qui composent l'ouvrage analysent cette tension récurrente entre l'existence d'espaces formellement démocratiques (élections, presse indépendante, société civile) et la capacité du régime à se maintenir malgré l'accroissement des mécontentements. En 2006, suite à l'assassinat du journaliste Norbert Zongo, Hilgers et Mazzocchetti écrivaient déjà : 
"Le paradoxe des régimes semi-autoritaires est que l'imaginaire trouve un espace abstrait de libération, mais les transformations politiques concrètes, elles, demeurent limitées. Cette tension peut conduire au sentiment que le politique est un espace caractérisé par I'"absence de possible" » (Hilgers et Mazzocchetti, 2006).

Dès lors, la question principale développée dans l'ouvrage est de savoir comment s'opèrent les oppositions de consciences et de pratiques, les insubordinations et les révoltes vis-à-vis du pouvoir dans un contexte où leur légitimité n'est pas remise en cause, mais où elles aboutissent rarement aux résultats espérés. Ce que montre le livre, c'est que nombre de ces mouvements de protestation sont disparates. Ils n'ont pas nécessairement pour finalité l'alternance et, encore moins, la prise ou le renversement du pouvoir. En même temps, cependant, les contributions soulignent que les transformations institutionnelles ont certains effets. Pour se maintenir au fur et à mesure des années, voire des décennies, le régime a été contraint de procéder à une relative ouverture. Les sentiments de frustration et de mécontentement, renforcés par un contexte de précarité économique, ont désormais plus de liberté pour s'exprimer. Si cela ne suffit pas, à court terme pour le moins, pour structurer des revendications collectives intersectorielles, cela indique néanmoins une évolution du rapport au politique.

"(Au croisement d'une) approche top down soulignant la nécessité
de créer des dispositifs institutionnels pour faciliter l'apprentissage
de nouveaux comportements et guider le changement social selon
une visée du bien commun [...] (et d'une) perspective bottom up
centrée sur la capacité des acteurs, y compris collectifs, à s'orienter
par eux-mêmes dans un ordre social en changement, à réguler et à
réorganiser collectivement leur monde commun dans un régime
d'incertitude " (Hilgers et Mazzocchetti, 2010).

L'ouvrage expose alors comment la capacité d'émettre des revendications collectives efficaces s'opère à travers un apprentissage lent, inscrit dans l'histoire, ainsi que par un changement du cadre institutionnel rendant possible l'expression structurée des revendications. La question est de voir comment, au-delà de l'absence d'alternance au sommet de l'Etat, la reconfiguration institutionnelle élargit, même de manière involontaire, l'espace politique et contribue à transformer la perception du pouvoir dans le chef de ceux qui le subissent plus qu'ils ne l'exercent. 
Cette analyse originale des régimes " semi-autoritaires " résulte de la rencontre de deux pensées. La question des imaginaires et de l' " absence de possible " avait été explorée par Jacinthe Mazzocchetti (2009), alors que Mathieu Hilgers se consacrait à la nature de l'espace public, notamment par la publication de son magistral ouvrage : Une ethnographie à l'échelle de la ville. Urbanité, histoire et reconnaissance à Koudougou (Burkina Faso) (2009b). Un ouvrage programmatique dans lequel il exposait les thèses auxquelles il allait donner de l'ampleur ultérieurement. Dans cette monographie consacrée à la ville de Koudougou au Burkina Faso, Mathieu Hilgers explique que l'Afrique s'urbanise rapidement et que l'émergence des "villes moyennes " devient un enjeu majeur. Que signifie étudier une ville dans l'Afrique d'aujourd'hui et comment le faire ? L'anthropologue détaille les conditions d'une ethnologie de la ville moyenne. Qu'est-ce que l'urbanité et quelles sont les conditions pour une ethnologie urbaine ? Pour y parvenir, Mathieu Hilgers décrit la formation progressive d'une " identité urbaine partagée " qui force chaque habitant ou chaque groupe $d$ 'habitants à se positionner sur la scène urbaine et démontre comment, par ce mécanisme, les groupes de populations qui composent la ville, l'intègrent.

L'anthropologie des villes moyennes proposée par Mathieu Hilgers articule trois dimensions : I'urbanité, l'autochtonie et la réputation de la ville. Ici Koudougou identifiée au Burkina Faso comme une "ville rebelle » depuis la période coloniale française.

La première dimension est celle de "l'injonction à l'urbanité " qui débouche sur un "style urbain ". Ce style agit comme l'affirmation d'une nouvelle identité personnelle. La ville de Koudougou se décline par rapport aux villages environnants lorsque les ruraux qui y débarquent doivent l'apprivoiser. Dans des pages superbes, l'auteur explique comment chaque personne qui aborde la ville doit l'incorporer pour l'habiter. Sous cet angle, la ville équivaut à une manière d'être urbain qui demande une nouvelle domestication du corps.

La seconde dimension porte sur la notion d'autochtonie, décrite comme le rapport des citadins à l'histoire urbaine. Les rapports à l'autochtonie sont abordés sous l'angle de l'affrontement de différents registres de vérités, complexifiant singulièrement l'administration de la 
preuve et réduisant d'autant les chances d'aboutir à un accord partagé. La démonstration est servie par un admirable travail qui traite du déplacement des autels de la terre (les tengsés) d'un quartier à l'autre de la ville. Ces autels sont autant de ressources cultuelles qui circulent entre quartiers et familles en lutte pour défendre leur suprématie et entre partis politiques en compétition pour le pouvoir communal. L'analyse des enjeux fonciers conduit l'auteur à décrire une " coutume foncière urbaine " en acte. Être " apprécié comme autochtone » permet de prétendre à la terre urbaine. La compétition entre quartiers et familles concerne la primoarrivée plutôt que l'autochtonie proprement dite. Les récits de fondation des quartiers qui composent aujourd'hui Koudougou sont en compétition pour établir qui était le premier, avec comme enjeu l'accès aux droits fonciers actuels. Avec un sens aigu de l'observation, Mathieu Hilgers démontre comment ces " récits de fondation " renvoient surtout à la « production de localité " et à des usages politiques et symboliques : les " communautés fondatrices " sont mobilisées dans les constructions sociales actuelles, parties prenantes du débat public et de l'accès à la terre urbaine.

La troisième dimension traite de la réputation de la ville : « Koudougou, ville rebelle ". II est question d'une analyse passionnante et inédite des rapports des habitants, des responsables communaux et des partis politiques à l'identité de la ville, en particulier lors de l'assassinat du journaliste Norbert Zongo (natif de Koudougou). Koudougou, situé en marge des royautés mossi, joue un rôle important après l'indépendance dès lors que la ville donnera à la Haute-Volta son premier président. Cette ville, dont émergeront des dirigeants notoires de la période révolutionnaire sankariste, sous le régime de Blaise Compaoré est oubliée, punie par le parti au pouvoir. Koudougou, la rebelle, apparaît comme une construction sociale et une identité partagée, assignée.

De ces trois dimensions, il ressort que la méthode de recherche inventée par Mathieu Hilgers pour décrire la ville moyenne permet de montrer comment les expériences communes de la ville s'assemblent pour établir une véritable matrice pratique et représentationnelle partagée par les habitants. Ces représentations sociales constituent un espace d'articulation qui permet à différentes situations et à différents positionnements de prendre pied sur un espace d'influence. Sous certaines condi- 
tions, les enjeux autour du collectif d'appartenance débouchent sur des prises de parole contradictoires : il est alors question d'analyser les conditions de l'émergence de ces débats, ainsi que de l'exercice du politique.

La force du " collectif d'appartenance " est qu'il débouche sur une vision globale de la ville : " c'est un opérateur qui subsume de multiples identités sous une commune appartenance ". La ville acquiert un sens propre, fruit d'une identité discutée par ses habitants et reconnue à l'extérieur que ce soit par les chefferies, les autres villes, l'État, à l'occasion d'enjeux politiques régionaux et nationaux, voire internationaux lorsque la coopération au développement est impliquée.

Par sa pertinence et son acuité, cette recherche magistrale entreprise par Mathieu Hilgers (2009b), entrera rapidement en débat avec le champ de l'anthropologie politique et urbaine. Le génie de l'anthropologue a consisté ici à identifier les principes structurants de la réalité urbaine, conduisant ainsi à ouvrir la voie à une " anthropologie des villes moyennes". Dotées du sens de leur histoire, ces petites villes émergentes deviennent des acteurs dans un monde globalisé. Le concept de " collectif d'appartenance " restitue une capacité d'agir aux collectifs humains : il dégage la spécificité d'un espace de vie pratique, habité par un collectif, malgré les mouvements d'homogénéisation à l'œuvre et les tensions vécues par les groupes de personnes qui le composent. Par le concept de " collectif d'appartenance ", la ville moyenne apparaît dans sa globalité et sa singularité, servie par la mise en œuvre d'une ethnologie intégrative. Ce concept s'impose comme une évidence lorsqu'il assigne une identité singulière aux habitants d'une ville qui prend ainsi pied dans le jeu plus vaste de la reconnaissance. Mathieu Hilgers soutenait une interprétation en termes de réseaux d'acteurs, intégrée à la production d'un savoir global, toujours ethnographiquement situé.

En conclusion de ce texte, nous ne pouvons qu'avoir une pensée pour les enfants de Mathieu et de Géraldine. Sans boule de cristal, sans gris-gris, dans le flou, dans le trouble, nos questions s'entrechoquent et restent sans réponse. Nous n'avons rien à dire de ce choix de quitter la scène avant que "la fête ne soit finie ", " de ce rideau tombé » en cours de parcours, si ce n'est le miroir qui nous est tendu. D'un côté, être fidèle à cette anthropologie engagée que Mathieu Hilgers pratiquait et appelait de ses vœux ; 
garder toujours à l'esprit l'exigence de rigueur tout en ne lâchant rien, jamais, des défis éthiques. De l'autre, la nécessité de se recentrer sur l'essentiel, en dehors des diktats de ce monde néolibéral et de la pensée managériale qui l'accompagne, en dehors également de cette course éperdue que le monde universitaire nous impose, nous enjoignant à toujours plus de productivité, peu en importe le prix humain tant que les rankings s'envolent.

\section{Bibliographie}

ANDRE G. et HILGERS M. (éds.), 2009, " Intimités et inimitiés du religieux et du politique en Afrique ", Civilisations, 58/2.

HILGERS M., 2007, "Objectivation participante ", in CAZIER J.-P., ABCédaire Pierre Bourdieu, Sil Maris-Vrin : 132-134.

HILGERS M., 2009a, " Habitus, Freedom and Reflexivity ", Theory and Psychology, 19/6: 728-755.

HILGERS M., 2009b, Une ethnographie à l'échelle de la ville. Urbanité, histoire et reconnaissance à Koudougou (Burkina Faso), Paris, Karthala.

HILGERS M., 2010, "The Three Anthropological Approaches to Neoliberalism », International Social Science Journal, 62/201: 351-363.

HILGERS M., 2011a, "Autochtony as a Capital in a Global Age ", Theory, Culture and Society, 28/1: 34-54.

HILGERS M., 2011b, " L'autochtonie comme capital: appartenance et citoyenneté dans l'Afrique urbaine», Anthropologie Sociale-Social Anthropology, 19/2 : 143-158.

HILGERS M., 2011c, « L'autochtonie en milieu urbain Ouest Africain. Eléments pour une approche comparative ", in JUL-LARSEN E., LAURENT P-J., LE MEUR $\mathrm{P}-\mathrm{Y}$. et LEONARD E., Une anthropologie entre pouvoirs et histoire. Conversations autour de l'ouvre de Jean-Pierre Chauveau, Paris, Karthala : 383-404.

HILGERS M., 2012, " The Historicity of the Neoliberal State ", Social Anthropology, 20/1: 80-94. 
HILGERS M., 2013a, "Bourdieu, Pierre ", in McGEE R.J. et WARMS R.L., Theory in Social and Cultural Anthropology: an Encyclopaedia, Los Angeles, Sage: 89-94.

HILGERS M., 2013b, " Embodying Neoliberalism: Thoughts and responses to critics ", Social Anthropology, 21/1: 75-89. "Answer to Jamie Peck, Nik Theodore, Stephen Collier, Daniel Goldstein, Johanna Bockman, Don Kalb and Bob Jessop who commented the debate on neoliberalism engaged by Wacquant and Hilgers ", Social Anthropology 2012, 20/2 et 3, 2013, $21 / 1$.

HILGERS M. et MANGEZ E. (éds.), 2014, Bourdieu's Theory of Social Field: Concepts and Application, London, Routledge.

HILGERS M. et MAZZOCCHETTI J. (éds.), 2006, " Le Burkina Faso : I'alternance impossible ", Politique africaine, Karthala : 101.

HILGERS M. et MAZZOCCHETTI J. (éds.), 2010, Révoltes et oppositions dans un régime semi-autoritaire. Le cas du Burkina Faso, Paris, Karthala.

MAZZOCCHETTI J., 2009, Etre étudiant à Ouagadougou. Itinérances, imaginaire et précarité, Paris, Karthala.

OTTAWAY M., 2003, Democracy Challenged: The rise of semi-authoritarianism, Washington, Carnegie Endowment for International Peace.

\title{
ANiviv
}

\author{
Pierre-Joseph Laurent est Professeur d'anthropologie \\ Université catholique de Louvain-la-Neuve \\ Email : pierre-joseph.laurent@uclouvain.be \\ Jacinthe Mazzocchetti est Professeure d'anthropologie \\ Université catholique de Louvain-la-Neuve \\ E-mail : jacinthe.mazzocchetti@uclouvain.be
}

